\title{
Self-propelled droplets
}

\author{
Ralf Seemann ${ }^{1,2, a}$, Jean-Baptiste Fleury ${ }^{1, b}$, and Corinna C. Maass ${ }^{2, c}$ \\ 1 Experimental Physics, FR 7.2, Saarland University (UdS), Saarbrücken, Germany \\ 2 Max Planck Institute for Dynamics and Self-Organization, Göttingen, Germany
}

Received 24 February 2016 / Received in final form 20 April 2016 Published online 10 November 2016

\begin{abstract}
Self-propelled droplets are a special kind of self-propelled matter that are easily fabricated by standard microfluidic tools and locomote for a certain time without external sources of energy. The typical driving mechanism is a Marangoni flow due to gradients in the interfacial energy on the droplet interface. In this article we review the hydrodynamic prerequisites for self-sustained locomotion and present two examples to realize those conditions for emulsion droplets, i.e. droplets stabilized by a surfactant layer in a surrounding immiscible liquid. One possibility to achieve self-propelled motion relies on chemical reactions affecting the surface active properties of the surfactant molecules. The other relies on micellar solubilization of the droplet phase into the surrounding liquid phase. Remarkable cruising ranges can be achieved in both cases and the relative insensitivity to their own 'exhausts' allows to additionally study collective phenomena.
\end{abstract}

\section{Introduction}

The emergence of "living patterns" of self-propelled biological objects is well known from large animals like flocks of birds or schools of fish, but also from small biological animals like armies of ants or even simple objects like bacterial colonies. Similarly to the large differences in size, the interactions between individual elements differ remarkably as well. The interactions can vary from purely physical, as hard core repulsion or hydrodynamic interactions, to more complex interactions like visual or chemical sensing. As many of the observed swarming behaviors obey similarities this raises the question of a fundamental understanding of the basic mechanisms of locomotion and pattern formation of self-propelled objects. As none of the aforementioned biological self-propelled objects are free from biochemical sensing there is a large interest in artificial systems with well controlled chemical and physical interactions, which can be modeled precisely in a physical framework. To reduce complexity in the model systems it is desired that the self-propelled objects do not interact strongly with any kind of chemical product left behind in their traces, and the propulsion should be long lived, allowing the system to reach non-equilibrium steady states of

\footnotetext{
a e-mail: r.seemann@physik.uni-saarland.de

b e-mail: jean-baptiste.fleury@physik.uni-saarland.de

c e-mail: corinna.maass@ds.mpg.de
} 
collective behavior. A simple model system for those types of artificial swimmers are self-propelling droplets which can obey a well-controlled propulsion and a known flow field leading to controllable hydrodynamic interactions.

Various mechanisms for droplet locomotion have been already studied, e.g. by phase separation of binary mixtures driven by emerging hydrodynamic flow and non-diffusive transport [1,2]. Thakur et al. [3] studied phase separation at a nematic-isotropic transition of a thermotropic liquid crystal doped with $20 \mathrm{wt} \%$ of a nonmesogenic material. Other studies were concerned with binary liquid mixtures featuring a two-phase region in their phase diagram. In $[4,5]$ the binary system acetone-hexadecane and in $[6,7]$ mixtures of aqueous solutions of poly-(ethylene glycol) (PEG) and sodium sulfate $\left(\mathrm{Na}_{2} \mathrm{SO}_{4}\right)$ were studied. However, the droplets driven by phase separation dynamics show only short active periods and their estimated cruising range is typically below 20 times their diameter. Thus, those systems are less suited to serve as model systems for the study of collective behavior. Recently, it was also observed that liquid metal like EGaIn or Galistan can self-propel in an electrolyte (e.g. $\mathrm{NaOH}$-solution) when in contact with Aluminum flakes [8]. The resulting redox reaction stimulates a flow inside the liquid metal droplet which in turn propels the droplet in the surrounding electrolyte with a velocity of several droplet diameters per second. The cruising range of this system seems remarkable, but the reaction is accompanied by a trace of bubbles appearing next to the Aluminium flakes which is not desired for the study of collective behavior. In the following, we will concentrate in our review on two types of active emulsion droplets having the potential to study collective behavior and which were previously published in [9-11] and reviewed in a more general context in [12]. Both systems are driven by Marangoni stresses caused by a gradient in surface tension along the droplet interface. In one case the gradient is maintained by chemical reactions, in the other case by solubilization. The two different mechanisms lead to a similar self-propelling behavior that can be described as neutral swimmers using the squirmer model [13] and are therefore expected to show similar collective behavior. The propulsion velocities are of the order of some $10 \mu \mathrm{m} / \mathrm{s}$ and the cruising ranges are about $100-4000$ droplet diameters.

\section{Driving mechanism for self-propelled droplets}

\subsection{Marangoni effect}

The surface tension $\gamma$ has the dimension of an energy per area, respectively force per length. When the surface tension varies along an interface, its gradient has the dimension of a stress. In case of liquid/liquid interfaces this stress from surface tension gradients has to be balanced by viscous stresses in the liquids resulting in a viscous shear flow, which induces a current at the interface in the direction of increasing surface tension [14]. This Marangoni transport can be initiated by a concentration gradient, but also by temperature gradients, which is then called thermo-capillary effect. In a glass of strong wine the Marangoni effect can be easily observed by eye, cf. Fig. 1. The different evaporation rates of ethanol and water result in a concentration gradient of ethanol and thus to a surface tension gradient. The resulting stresses can lift the liquid upwards against gravity by some millimeters.

The Marangoni effect is particularly suitable for transport processes at small scales and small droplets. The order of magnitude of the velocity of a droplet with radius $R$ can be estimated from the tangential stress balance at the interface with dynamic viscosity $\eta=\eta^{o}=\eta^{i}$ being equal outside and inside the droplet $[15,16]$ :

$$
u \sim \frac{R \nabla \gamma}{\eta} .
$$



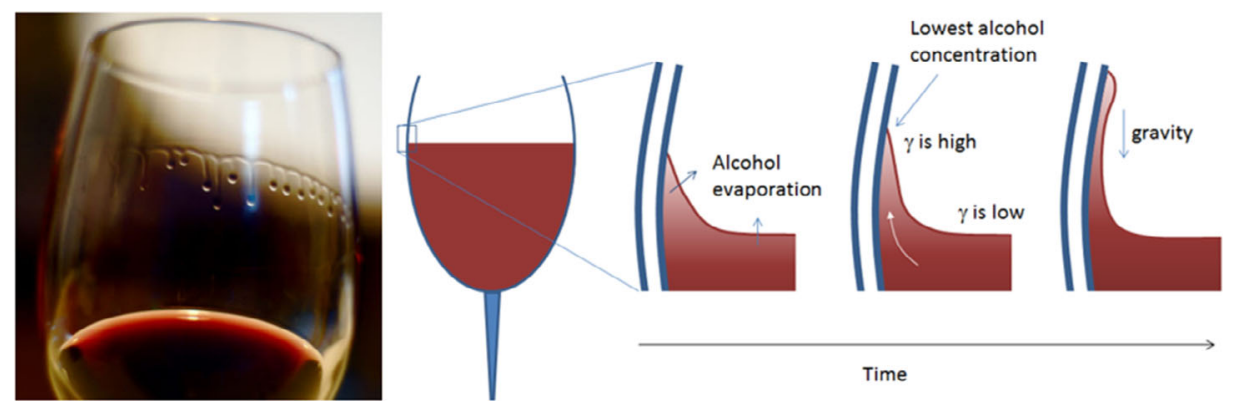

Fig. 1. (left) Tears of wine (photo from Benjamin Maples (2010), made in a course 'Flow Visualization' by Jean Hertzberg, University of Colorado, http://www.colorado. edu/MCEN/flowvis/course/). (right) Sketch how tears of wine are generated by the Marangoni effect, i.e. by a flow along the surface of two fluid phases (wine/air) caused by surface tension gradients along the interface between the two phases. (image credit: COMSOL; https://www.comsol.de/blogs/tears-of-wine-and-the-marangoni-effect/ This figure is subject to copyright protection and is not covered by a Creative Commons license.)

For a droplet with $R \approx 50 \mu \mathrm{m}$, a difference in surface tension of a surfactant decorated oil/water interface of about $\Delta \gamma \approx 10^{-3} \mathrm{~N} / \mathrm{m}$ across the droplet size (i.e. over a length of $100 \mu \mathrm{m}$ ) and viscosities $\eta$ on the order of one milli-pascal seconds, a typical velocity $u$ is of the order of $0.5 \mathrm{~m} / \mathrm{s}$. Hence, the Marangoni effect already provides sufficient drive at very small variations in surface tension, respectively for small differences in surfactant surface coverage.

\subsection{Flow field near a droplet}

The velocity field of a spherical droplet with radius $R$ driven by a homogeneous volume force and moving with velocity $V$ relative to the surrounding quiescent liquid phase is given by a solution of the Stokes equation with suitable boundary conditions. Besides the Laplace condition for normal stress component a continuity of the tangential stress is demanded at the surface. With the direction of motion as the polar axis, the solution of the Stokes equation can be written for the radial and the polar component in the rest frame of the droplet for the outer $(o)$ velocity field as [17]

$$
\begin{gathered}
v_{r}^{o}=V \cos \theta \cdot\left[-1+\frac{3+2 \mu}{2(1+\mu)} \frac{R}{r}-\frac{1}{2(1+\mu)}\left(\frac{R}{r}\right)^{3}\right] \\
v_{\theta}^{o}=-V \sin \theta \cdot\left[-1+\frac{3+2 \mu}{4(1+\mu)} \frac{R}{r}+\frac{1}{4(1+\mu)}\left(\frac{R}{r}\right)^{3}\right]
\end{gathered}
$$

and for the velocity field inside $(i)$ the droplet as

$$
\begin{aligned}
& v_{r}^{i}=V \cos \theta \cdot\left[-1+\frac{2+3 \mu}{2(1+\mu)}-\frac{\mu}{2(1+\mu)}\left(\frac{r}{R}\right)^{2}\right] \\
& v_{\theta}^{i}=-V \sin \theta \cdot\left[-1+\frac{2+3 \mu}{2(1+\mu)}-\frac{\mu}{(1+\mu)}\left(\frac{r}{R}\right)^{2}\right]
\end{aligned}
$$


a)

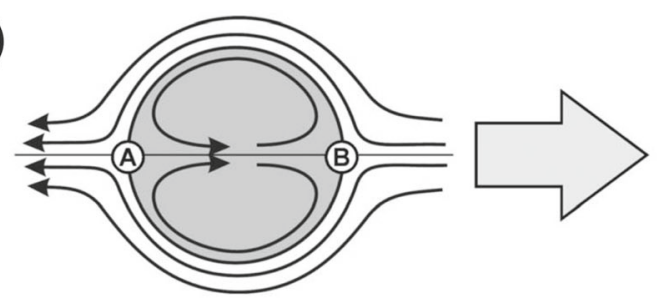

b)

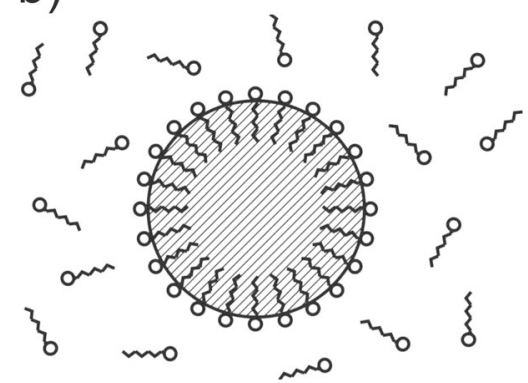

c)

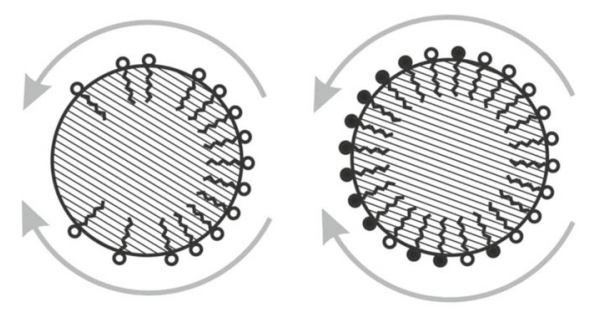

Fig. 2. a) Schematic of the flow field emerging inside and outside of a moving droplet shown in the co-moving frame of the droplet. The large arrow to the right indicates the motion of the droplet with respect to the surrounding liquid. A and B denote stagnation points. b) Surfactant molecules adsorb at the droplet surface reducing the interfacial tension, $\gamma$. The surface coverage is in equilibrium with the surfactant concentration in the solution. c) Two possibilities to generate Marangoni stresses along the surface of a droplet: (Left) Inhomogeneous surfactant coverage, and (Right) a spatially constant surfactant coverage with spatially varying surface activity. Black/white surfactant denote lower/higher surface activity which might be caused by chemical reactions. In both cases, the resulting Marangoni stress points in the direction of increasing surface tension (arrows around the drop), the resulting droplet motion points into the opposite direction. (Reproduced from [11] - Published by The Royal Society of Chemistry. This figure is subject to copyright protection and is not covered by a Creative Commons license.)

Here, $\mu=\eta^{i} / \eta^{o}$ is the viscosity ratio of the liquid inside, $\eta^{i}$, and outside of the droplet, $\eta^{o}$. The velocity of the flow field decays algebraically with increasing distance from the droplet interface. The corresponding flow field with a convection roll inside the droplet and with two stagnant points at the poles of the droplet and a maximum velocity at the equator of the droplet is sketched in Fig. 2(a).

The droplets considered so far have an isotropic surface without internal stresses. However, in case of a surface tension $\gamma$ varying along the droplet surface, e.g. due to an inhomogeneous surfactant coverage, a surface stress $\sim \nabla \gamma$ results. This surface (Marangoni) stress has to be balanced by viscous stresses which are caused by a Marangoni flow tangential to the droplet surface. Thus the continuity of the tangential stress between the outer and inner bulk fluids in presence of Marangoni stresses at the surface has to be replaced by $[18-20]$

$$
\tau_{r \theta}^{o}-\tau_{r \theta}^{i}=\frac{1}{R} \frac{\mathrm{d} \gamma}{\mathrm{d} \theta}
$$

The reader is referred to [20-22] for a more complete force balance for a Newtonian interface accounting for the effects of dilatation, shear and surface viscosities. 
Using Eq. (6) as additional boundary condition to the general polynomial solutions an expression for the interfacial velocity reads $[19,23]$

$$
\left.u_{\theta}\right|_{r=R}=\frac{V}{2} \frac{\eta^{o}}{\eta^{i}+\eta^{o}} \sin \theta+\sum_{n=2}^{\infty} \frac{n(n-1)}{2\left(\eta^{i}+\eta^{o}\right)}\left[\int_{0}^{\pi} C_{n}^{-1 / 2}\left(\cos \theta^{\prime}\right) \frac{\mathrm{d} \gamma}{\mathrm{d} \theta^{\prime}} \mathrm{d} \theta^{\prime}\right] \frac{C_{n}^{-1 / 2}(\cos \theta)}{\sin \theta}
$$

Here, $C_{n}^{-1 / 2}$ is the Gegenbauer polynomial of order $n$ and degree $-1 / 2$.

The adsorption of surfactant to, and the desorption of surfactant from the interface, either from the inner or from the outer phase, is estimated by an equilibrium between interface and surfactant containing phase by a linear Gibbs isotherm,

$$
\Gamma=\frac{-c}{k_{B} T} \frac{\mathrm{d} \gamma}{\mathrm{d} c} \equiv K c
$$

with $c$ denoting the bulk and $\Gamma$ the interfacial concentration of individual surfactant molecules. The transport of surfactant is described by a convective diffusion equation $[19,20]$

$$
\boldsymbol{u} \cdot \nabla c=\frac{2}{\mathrm{Pe}} \nabla^{2} c
$$

The relative strength of advective versus diffusive transport is taken into account by the Péclet number $\mathrm{Pe}=2 R V / D$ with $D$ being the diffusion coefficient.

This feedback mechanism can only be approximated or solved numerically. For a droplet moving through a surfactant containing liquid phase it means qualitatively that the distortion of the interface by the flow field will result in an accumulation of surfactant molecules at the trailing pole of a droplet. Such an inhomogeneous distribution of surfactant in turn will cause a Marangoni stress toward the leading pole of a droplet. Diffusion in the interface and ad-/desorption of surfactant will additionally counteract a potential gradient in interfacial tension. Thus, without external forcing, the droplet is driven toward an equilibrium state of no motion and constant surfactant coverage, cf. Fig. 2(b,c).

To propel droplets by Marangoni stresses and to achieve a steady motion the above described effect has to be inverted by alternative strategies to maintain a surface tension gradient. In the following section we will discuss how a surface tension gradient that enables propulsion, can be obtained by either a chemical reaction which transforms the surfactant into another kind of surfactant with different surface activity (Sect. 3) or by a concentration gradient in the surrounding liquid (Sect. 4).

\section{Schemes to utilize chemical reactions}

Several examples of chemical reaction schemes can be found in literature [9-11,24-31]. The common feature of all these systems is that the used surfactants are affected by a chemical reaction and the surface tension of an interface covered with the pristine surfactant differs from the surface tension of an interface covered with surfactant after the chemical reaction.

\subsection{Experimental realization \& properties of self-propelling droplets utilizing chemical reactions}

As an example we will explain the experiments presented in [9-11] in some more detail: Aqueous droplets having a diameter of about 100 micron are prepared in squalane 

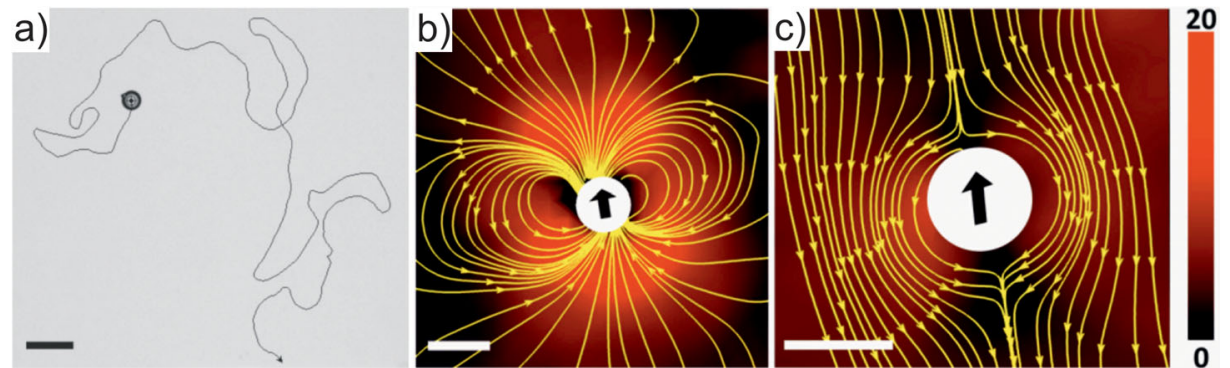

Fig. 3. Behavior of a single self propelled droplet being confined to a quasi-2D space between two hydrophobic glass plates. (a) Flow path of a droplet, scale bar is $300 \mu \mathrm{m}$. (Reproduced from [9] (http://dx.doi.org/0.1088/1367-2630/13/7/073021), C IOP Publishing \& Deutsche Physikalische Gesellschaft. CC BY-NC-SA.) (b) \& (c) Velocity fields around a droplet as determined by PIV reveal a flow field similar to that of a neutral squirmer in 3D. Yellow lines denote stream lines of the flow. (Reproduced from [11] - Published by The Royal Society of Chemistry. This figure is subject to copyright protection and is not covered by a Creative Commons license.) The magnitude of the velocity is color coded in microns per second, scale bars denote 100 microns. (b) Velocity field in the laboratory frame and (c) in co-moving droplet frame after subtracting the droplet velocity.

containing the surfactant mono-olein (MO). The concentration of MO is well above the critical micelle concentration (CMC). A mixture of $50 \mathrm{mM}$ sulphuric acid, $28 \mathrm{mM}$ sodium bromate, $400 \mathrm{mM}$ malonic acid, and $2.7 \mathrm{mM}$ ferroin is added to the droplet phase which continuously releases bromine. When the released bromine reaches the surface of the droplet it will react with the $\mathrm{C}=\mathrm{C}$ double bonds in the hydrophobic tails of the $\mathrm{MO}$ molecules. As a result of this bromination reaction the surface active properties of MO are changed. The interfacial tension of a water/squalane interface covered with pristine $\mathrm{MO}$ is $1.3 \mathrm{mN} / \mathrm{m}$ and the interfacial tension for brominated $\mathrm{MO}$ (brMO) is about $3 \mathrm{mN} / \mathrm{m}$.

The thus prepared aqueous droplets in squalane spontaneously self-propel. The propulsion velocity is on the order of a few microns per second and appears to be insensitive to the exact size of the droplets. The velocity of the droplet motion is decaying in time and the droplet motion finally stops after several minutes. The trajectory of an individual droplet is shown in Fig. 3(a) with a total running time of $400 \mathrm{~s}$ and a velocity of about $15 \mu \mathrm{m} / \mathrm{s}$. The trajectory is reminiscent of a random walk with a persistence length exceeding the size of the droplet, i.e. much larger than expected for Brownian motion. It is important to note that the propelling droplet can cross its own path. This is at variance to other self-propelling mechanisms that alter the continuous liquid phase in a way that self-crossing of the path is prevented [32]. Because of this property, the type of Marangoni driven self-propelling droplets presented above are well suited to explore collective motion.

Using particle image velocimetry (PIV), the flow profile around self-propelling droplets can be obtained. For PIV measurements micron sized fluorescent tracers are added to the liquid phase that follow the flow without delay. Two fluorescent microscopy images are captured with a defined time interval and the respectively observed fluorescent patterns are correlated to obtain the flow field. A resulting flow field is displayed in Fig. 3(b) as directly obtained in the laboratory frame. Subtracting the droplet velocity from that flow field one obtains the flow profile in the reference frame of the droplet, cf. Fig. 3(c). The experimentally determined flow fields in a confined 2D geometry are similar to those expected for neutral squirmer in 3D [13].

The droplet velocity and the cruising range are shown in Fig. 4(a) as a function of surfactant concentration. The CMC for this oil/surfactant system is $\sim 1 \mathrm{mM}$, so for all displayed data points the vast majority of the $\mathrm{MO}$ is present in micellar form. 
a)

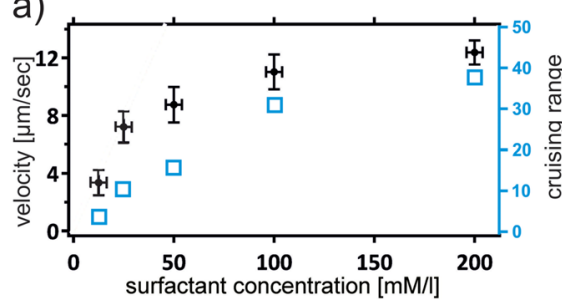

b)

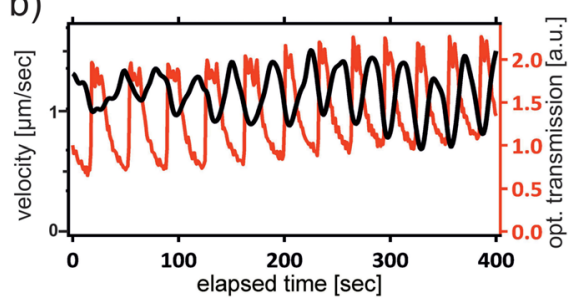

Fig. 4. (a) Velocity (black dots) and cruising ranges (blue open squares) of self-propelling droplets as a function of mono-olein concentration. The cruising range is given in units of one droplet diameter; every data point is an average of 50 separate droplets with diameter $=80 \mu \mathrm{m}$. (b) Locomotion speed (black) and optical transmission (red) as function of time recorded for droplets filled with oscillating BZ-solution. (Reproduced from [11] - Published by The Royal Society of Chemistry. This figure is subject to copyright protection and is not covered by a Creative Commons license.)

The swimmer velocity increases with MO concentration and plateaus at large MO concentrations. A similar behavior is observed for the cruising range, which only increases slightly for large $\mathrm{MO}$ concentrations. The plateau for the droplet velocity might be a result of the disintegration time of micelles at the droplet surface, which does not depend on the $\mathrm{MO}$ concentration and becomes the rate limiting step at large $\mathrm{MO}$ concentrations. The insensitivity of the droplet velocity to the MO concentration is a highly desired property when exploring their collective behavior. During the ongoing bromination reaction, the concentration of brMO in the oil phase increases inhomogeneously, due to the exhausts of the propelling droplets present in the system. As most of the brMO will be concentrated in micelles, the main result of the increasing brMO concentration is to effectively reduce the MO concentration in the surrounding squalane. Thus, there is hardly any dependence of the droplet velocity on the brMO concentration when conducting experiments at sufficiently large MO concentrations and unwanted interactions between propelling droplets or their trails can be minimized.

In contrast to the saturation of the swimmer velocity at high $\mathrm{MO}$ concentrations, the velocity depends sensitively on the availability of bromine that is maintaining the bromination of the MO. This dependence can be seen in Fig. 4(b), where the supply of bromine is varying with time. For this experiment, the previously used chemical mixture contained in the droplets yielding a constant bromine release is changed to one similar to the oscillating Belouzhov-Zhabotinsky-solution (BZ) [33,34] with an oscillating bromine release [9]. A particular feature of this reaction is that the oscillatory release of iron ions from the ferroin changes the color of the solution which allows to optically monitor the oscillations of the $\mathrm{BZ}$ reaction. The observed optical transmissions of the droplets are plotted together with their velocity in Fig. 4(b) and reveal that the droplets accelerate upon bromine release and decelerate when the bromine release is stopped.

A typical example of collective behavior of the self-propelling droplets in a quasi 2D geometry is shown in Fig. 5. The droplets have a flow field as shown in Fig. 3 similar to a neutral squirmer mode in 3D [13]. The arrows indicate the direction of motion of each droplet. After some time, long-lived clusters of different sizes are formed with distinct polar alignment of the velocities of neighboring droplets [9], cf. Fig. 5(b). This behavior is expected to change when the qualitative flow field changes e.g. from a neutral squirmer to a pusher or a puller. Besides the direction also the distance of interaction should change as the hydrodynamic interaction of a puller or pusher in $3 \mathrm{D}$ scales at $1 / r^{2}$ instead of $1 / r^{3}$ for a neutral swimmer. Thus collective 

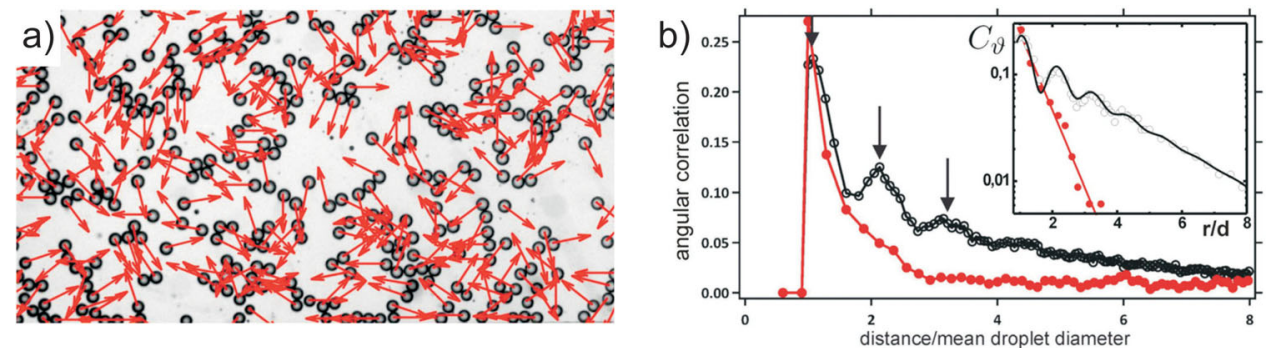

Fig. 5. (a) Optical micrograph showing collective droplet behavior; droplet diameter $d=80 \mu \mathrm{m}$, surfactant conc. $200 \mathrm{mM} / \mathrm{l}$. Arrows at the droplets indicate their current direction of motion. (b) Angular correlation function $C_{\vartheta}:=\left\langle\delta\left(r-\left|r_{i}-r_{j}\right|\right) \cos \vartheta_{i j}\right\rangle_{t, i j}\left(\vartheta_{i j}\right.$ is the angle between the droplet velocities) as function of distance of the droplet centers, $r / d$. The lower/upper curve describes the behavior for an area droplet density of 0.46 and 0.78 , respectively. The vertical arrows denote $r / d=1.08,2.16$ and 3.24 . The inset shows the same data in a semi-logarithmic plot. (Reproduced from [9] (http://dx.doi.org/ 0.1088/1367-2630/13/7/073021), (C) IOP Publishing \& Deutsche Physikalische Gesellschaft. CC BY-NC-SA.)

motion for pullers or pushers is expected to appear already at lower density than for neutral squirmers.

\subsection{Mechanism of locomotion utilizing chemical reactions}

For the droplet swimmers described in subsection 3.1, the self-propulsion originates from interfacial tension gradients. These gradients are caused by surfactant layers that are chemically modified, leading to a reduced surfactant activity. The resulting Marangoni stresses act against the interfacial tension gradients and impede the selfpropelled motion. To achieve self-propulsion, a mechanism is required to generate and maintain a sufficient counteracting interfacial tension gradient $\nabla \gamma$ in the direction of the droplet front. Such a mechanism has been modeled in several studies [23,35] via an advective transport of reactants from inside a droplet to its surface. The reactants modify the surfactant at the interface and with it the corresponding interfacial tension (see Fig. 2). To model this mechanism, the advection-diffusion-reaction contribution for each chemical component has to be included into the governing equations.

In the following we briefly summarize the study by Schmitt and Stark [23], who modeled a system where the surface activity is modified by advection of a chemical reactant from the inside. Without loss of generality, the authors refer to the specific experimental system discussed above and first published in Ref. [9]. The approach is similar to the one outlined in Eqs. (6)-(9) but Schmitt and Stark use an interfacial advection-diffusion equation accounting for the dynamics and chemical interaction of two different surfactant species, brominated and unbrominated, with concentrations $c_{1}$ and $c_{2}, c_{1}+c_{2}=1$, and the concentration difference $\phi=c_{1}-c_{2}$ as order parameter. The authors define the surfactant dynamics at the interface in terms of the concentration difference $\phi$, the time evolution of which is driven by the diffusive current $\boldsymbol{j}_{D}$ and the advective current $j_{A}$, and a simple linear reaction term approximating adsorption, desorption, and chemical conversion of brominated and unbrominated species:

$$
\dot{\phi}=-\nabla \cdot\left(\boldsymbol{j}_{D}+\boldsymbol{j}_{A}\right)-\tau_{R}^{-1}\left(\phi-\phi_{e q}\right) .
$$

They model the situation where the diffusive current counteracts a bromination gradient, $\boldsymbol{j}_{D} \propto-\boldsymbol{\nabla} \phi$, whereas the advective current is expected to reinforce it, $\boldsymbol{j}_{A} \propto \nabla \phi$. 
A kinetic instability propelling the droplet arises if the advective current prevails. The advective current, $\boldsymbol{j}_{A}=\phi \boldsymbol{u}$, is coupled to the velocity profile at the interface $\boldsymbol{u}$, having the form of Eq. (7). If advection dominates diffusion the system develops an instability. The authors observe four different states for active droplets: resting, swimming, oscillating, and a "stopping" state in which the droplet comes to rest after a short swimming period.

\section{Schemes to utilize phase transformations}

We now turn to a second type of surfactant stabilized droplets that show spontaneous locomotion. In this system, the surfactant layer stabilizing the droplets is modified by an inhomogeneous depletion that results from a solubilization of the droplet phase into a micellar nanoemulsion.

\subsection{Experimental realization \& properties of self-propelling droplets utilizing phase transformations}

At oil/water interfaces spontaneous convection driven by surface tension gradients frequently occurs in case of mass transfer [36]. This effect can be seen when partially water soluble droplets of e.g. pentanol [37] or aniline [38,39] are generated on the liquid/air interface of an aqueous phase. The droplet undergoes a spontaneous selfpropelled motion when its content dissolves in the surrounding aqueous phase and potentially splits into smaller droplets. A similar behavior can be found also if either the continuous or the droplet phase contains surfactants and the surface of the droplet is covered by surfactant $[40,41]$. If the liquids contain surfactant, the dissolution can be regarded as micellar solubilization and at the end of the process most of the droplet phase will be stored in micelles.

The process of micellar solubilization has been studied for aqueous phases and different combinations of organic droplets and surfactants [42] and convective flow patterns were found in some cases during solubilization $[43,44]$. The systematic observation of spontaneous droplet locomotion during solubilization is quite recent and so far only aqueous droplets in a mixture oil and nonionic surfactants [45] and organic droplets in an aqueous solution containing ionic surfactants $[11,47]$ have been studied in detail.

To shed some light to this propulsion mechanism we consider water droplets in a mixture of squalane and the surfactant MO, as was studied by [45]. The authors used a MO concentration $(25 \mathrm{mM})$ in squalane well above the CMC meaning that a significant amount of inverse MO micelles are present in the continuous squalane phase, cf. Fig. 6. In this solution aqueous droplets with a radius of some ten microns self-propel with initial velocities of some ten $\mu \mathrm{m} / \mathrm{s}$ that decrease with time until the droplet motion stops. Parallel to its motion, the droplet loses volume. However, no spontaneous droplet motion was found if either the MO concentration was below the $\mathrm{CMC}$ or if the squalane/MO solution was saturated with water. These findings indicate that, in fact, the key for the spontaneous droplet locomotion lies in aqueous droplets undergoing a micellar solubilization process in the squalane/MO solution. The results were interpreted in [45], extending a theory that was intended to model the autophoretic motion of colloidal particles [35, 46].

In the following we consider a second system that is based on the solubilization of nematic liquid crystal droplets (4-pentyl-4-cyanobiphenyl (5CB)) in aqueous surfactant solutions (ionic surfactant tetradecyltrimethylammonium bromide (TTAB)) $[11,47]$. The surfactant concentrations ranged from values around the CMC $(0.13 \mathrm{wt} \%$ 

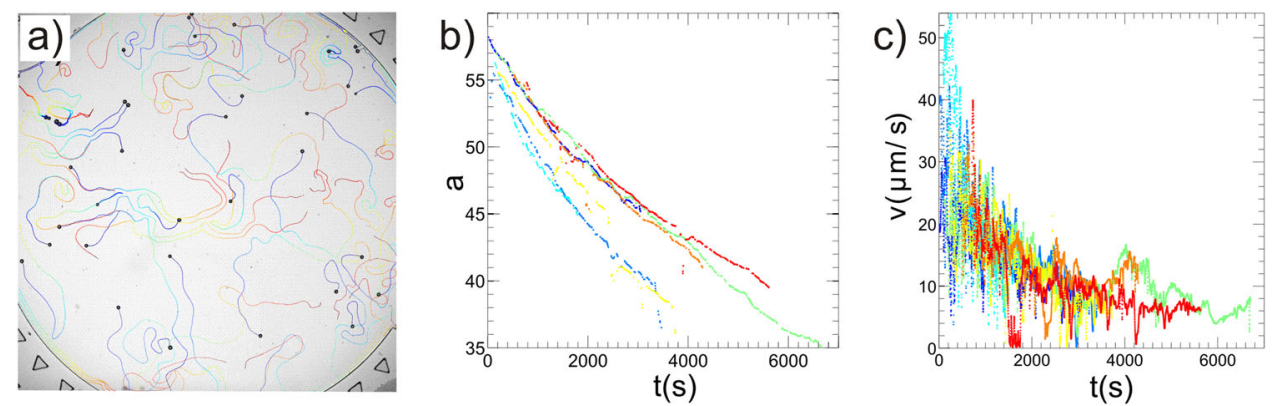

Fig. 6. Behavior of water droplets in a surrounding squalane phase with $25 \mathrm{mM}$ monoolein. (Left) Droplet trajectories recorded during $500 \mathrm{~s}$. (Middle) Droplet velocity as function of time (for 8 trajectories). (Right) Droplet diameter of active droplets as function of time. (Figure adapted with permission from Ref. [45], http://dx.doi.org/ 10.1103/PhysRevLett.113.248302 (C) (2014) by the American Physical Society).
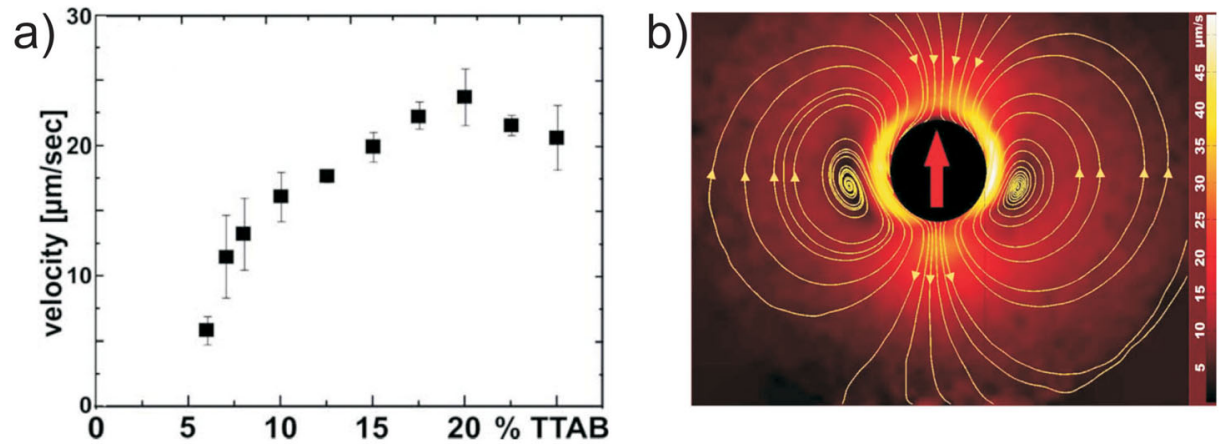

Fig. 7. Self-propelling $5 \mathrm{CB}$ droplets in an aqueous surfactant (TTAB) solution. (Left) Droplet velocity as function of TTAB concentration. (Right) Velocity field around a droplet in the laboratory frame as determined by PIV. The direction of droplet motion parallel to the bottom wall is indicated by the large arrow. Stream lines of the flow are shown in yellow color. The spiral pattern of the flow lines are presumably an artefact from the limited lateral PIV resolution, i.e. from the size of the interrogation window (Reproduced from [11] Published by The Royal Society of Chemistry. This figure is subject to copyright protection and is not covered by a Creative Commons license.)

of TTAB in water) to values well above the CMC (up to $25 \mathrm{wt} \%$ ). Similar to the aforementioned observations from [45], the radius of a $5 \mathrm{CB}$ droplet decreased linearly with time in the aqueous surfactant solution when the TTAB concentration was above the CMC, also suggesting a solubilization of the droplet phase [47]. Spontaneous selfpropulsion of the $5 \mathrm{CB}$ droplets was found when the TTAB concentration was larger than 5 wt\%, cf. Fig. 7(a). For larger surfactant concentrations both the volume loss and the droplet velocity increase (from $5 \mu \mathrm{m} / \mathrm{s}$ to about $25 \mu \mathrm{m} / \mathrm{s}$ ).

By tracking the movement of small textural features in a $5 \mathrm{CB}$ droplet, a convective flow field can be observed inside the droplet resembling the flow field expected from Eqs. ((4) \& (5)). The velocity field around a self-propelling 5CB droplet is shown in Fig. 7(b) which is comparable to the flow field found for the self-propelling droplets driven by the bromination reaction discussed above (cf. Fig. 3), being also reminiscent of the flow field expected for neutral squirmers.

In sufficiently dense droplet ensembles, the hydrodynamic interaction between droplets results in collective phenomena, cf. left panel of Fig. 8. In the system as described above, without density matching, the 5CB droplets typically form rafts, 

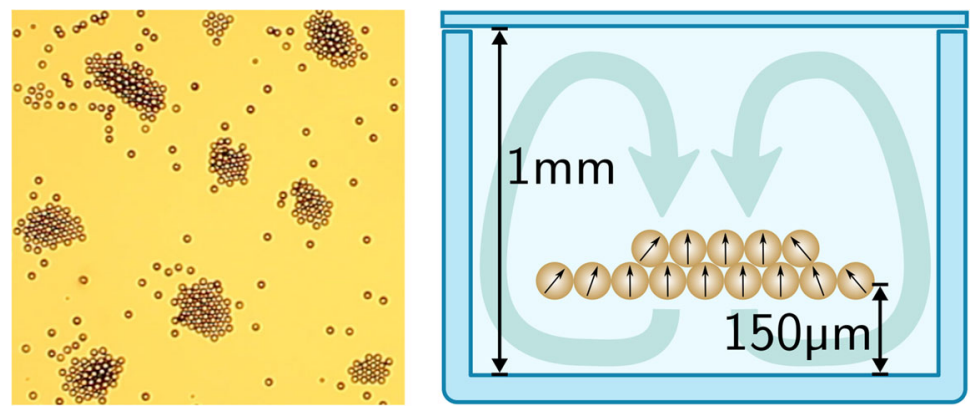

Fig. 8. $5 \mathrm{CB}$ droplets forming clusters in a $3 \mathrm{~d}$ microfluidic cell. (Left) Optical micrograph, droplets are $50 \mu \mathrm{m}$ in diameter. (Right) Convection roll formed around a stable droplet cluster sketched by blue arrows. Reservoir height $H \approx 1 \mathrm{~mm}$, distance of droplet cluster to bottom $h \approx 0.15 \mathrm{~mm}$. Symmetry axis (of self-propelled motion) of droplets is indicated by black arrows.

lift off from the bottom and float close to the bottom of the microfluidic cell. Such a floating raft is surrounded by a convection roll, as sketched in the right panel of Fig. 8. Conducting these experiments in a microfluidic cell with height well above the diameter of a single droplet, the system undergoes a clustering transition even if there are only a few droplets in the cell. In contrast the droplet clustering is absent even at large droplet densities if the densities of the droplets and surrounding aqueous phase are matched and the droplets do not sediment. In this 3D situation, there is no possibility for the droplets to form such a convection pattern, and the droplets just escape into the third dimension. Thus one can conclude that the observed clustering is governed by the formation of a large scale convection roll and also that these large scale flow patterns are determining the collective droplet behavior in addition to the motion of single particles [48].

\subsection{Mechanism of locomotion utilizing phase transformations}

In the cases of the aqueous droplets in squalane/monoolein solution, cf. Fig. 6 \& Ref. [45], and the 5CB droplets in an aqueous TTAB solution, cf. Figs. 7, 8 \& Ref. [11], the Marangoni stresses driving the self-propelled droplet motion cannot result from a chemical reaction modifying the surfactant molecules at the droplet interface. Heterogeneities in the surfactant layers of the droplets are rather expected to result from the solubilization process. In the following paragraphs, we will discuss how solubilization can deplete the surfactant molecules in a region close to the droplet surface. Spontaneous motion of a droplet can cause a symmetry breaking of the depleted region resulting in a Marangoni stress that maintains the droplet motion. For the sake of simplicity this discussion will be restricted to the $5 \mathrm{CB}$ droplets in an aqueous surfactant solution.

Oils solubilize in aqueous surfactant solutions via two mechanisms [42,49]: the micellar pathway, where micelles collect oil molecules directly at the oil-water interface, and the molecular one, where the oil molecules are picked up a certain distance away from the interface, Fig. 9. It is important to note that for both processes the surfactant covered droplet surface is depleted of surfactant molecules as filled micelles contain more surfactants than unfilled micelles. However, in the case of the $5 \mathrm{CB} / \mathrm{TTAB}$ system the latter pathway is the preferred one, as the charged TTAB micelles are repelled from the interface and $5 \mathrm{CB}$ is water soluble to a certain extent and thus able to diffuse into the aqueous phase. 
micellar pathway

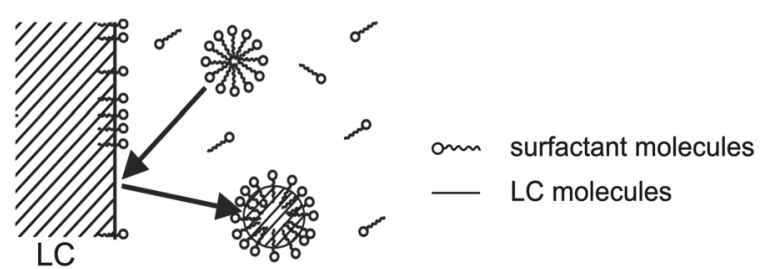

molecular pathway

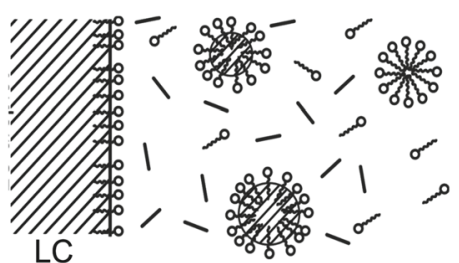

Fig. 9. Schematic of micellar and molecular solubilization pathways. The liquid crystal (LC) is contained in the droplets surrounded by a micellar surfactant solution. (Left) Micelles can be filled with LC when they touch the droplet surface (micellar pathway). (Right) LC molecules diffusing in the surrounding phase can be collected in micelles (micellar pathway). (Reproduced from [11] - Published by The Royal Society of Chemistry. This figure is subject to copyright protection and is not covered by a Creative Commons license.)
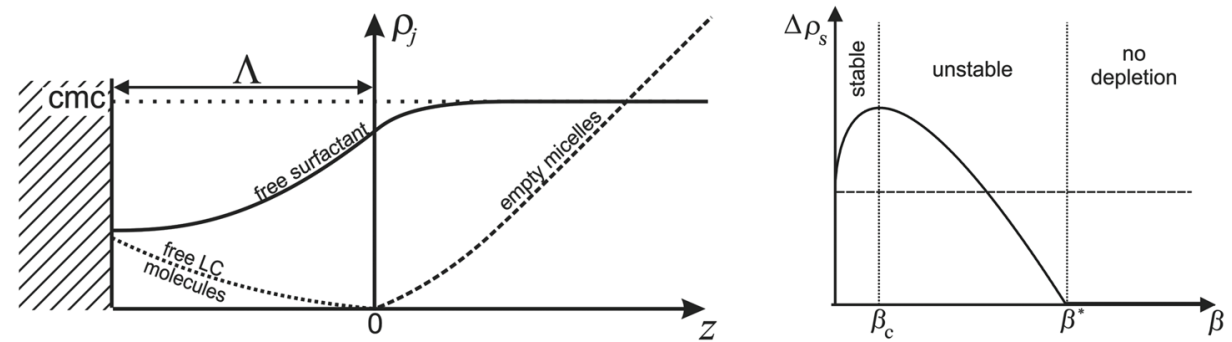

Fig. 10. Qualitative illustration of surfactant concentration and depletion for the molecular pathway. (Left) Surfactant concentration profiles in the diffusive steady state. $\Lambda$ is the extension of the region around a droplet where micelles can get filled. (Right) Surfactant depletion in the vicinity of a droplet surface as function of the driving parameter $\beta$. Whether a droplet moves spontaneously (unstable situation) depends on the slope of the curve. For $\beta>\beta_{c}$, there is no depletion and the surface of a droplet is fully covered with surfactants. (Reproduced from [11] - Published by The Royal Society of Chemistry. This figure is subject to copyright protection and is not covered by a Creative Commons license.)

As micelles grow in size while imbibing oil molecules, the density $\rho_{s}$ of free surfactant molecules in the continuous phase, which equals the critical micelle concentration (CMC) at equilibrium, is depleted, as well as, in consequence, the surfactant coverage of the interface by a value of $\Delta \rho_{I}$. The disintegration of empty micelles drives $\rho_{s}$ back towards the CMC equilibrium, subject to the empty micelle density $\beta$. Considering that empty micelles are advected at the leading pole of a moving droplet, taking up oil while travelling past the droplet, the surface depletion $\Delta \rho_{I}$ is less pronounced near the leading pole due to the higher empty micelle availability $\beta$. This results in Marangoni stresses pointed towards the base of the droplet, propelling it forward.

This process can be approximated qualitatively considering the diffusive behavior of empty and filled micelles, free surfactant and oil molecules [11], establishing two characteristic length scales: $\lambda \propto \beta^{-1 / 3}$, denoting the range of surfactant depletion due to micellar filling, and $\Lambda \propto \beta^{-1}$, characterizing the free diffusion of the oil molecules until they are collected by empty micelles. Qualitatively, it follows that

$$
\Delta \rho_{I}=c_{1} \beta^{2 / 3}+c_{2}\left(1-c_{3} \beta\right)
$$

Locomotion is sustained by surface depletion if $\partial \Delta \rho_{I} / \partial \beta<0$, corresponding to the range of $\beta_{c}<b<\beta^{*}$ in Fig. 10. 


\section{Conclusion}

Due to the motion dependent surfactant depletion of an emulsion droplet, a spontaneous motion of an emulsion droplet is damped under normal circumstances by a back flow induced by Marangoni stresses. However, spontaneous self-propelled droplet motion by Marangoni stresses can be sustained by either chemical reactions changing the activity of the surfactant molecules or by micellar solubilization of the droplet phase. For the first example, the bromination reaction of the surfactant molecule mono-olein was reviewed. The bromine is constantly supplied by a chemical mixture similar to a non oscillating Belouzhov-Zhabotinsky-solution and the spontaneous motion stops when either the release of bromine or the concentration of pristine surfactant molecules is fading. The second type of self-propelled motion, driven by micellar solubilization, relies on a sufficient concentration of surfactant molecules that needs to be above the CMC and a finite solubility of the droplet phase in the surrounding medium. The latter is needed to allow the surfactant micelles to pick up individual molecules from the droplet phase in the near vicinity of a droplet, as direct filling of micelles at the droplet surface cannot sustain the motion. The motion stops when either the surrounding medium is completely saturated with the droplet phase or the droplet phase is completely solubilized in the surrounding medium. Both realizations exhibit flow fields in the scope of the confining 2D geometry that are comparable to the flow fields of neutral squirmers in 3D. The droplets in both cases are fairly insensitive to their own changes in the surrounding medium allowing to explore collective phenomena. For the chemical reaction it was shown that droplets can form living rafts with an angular correlation that increases with increasing droplet density. In the case of solubilization it was observed that droplets can form rafts even at low density when confined in a 3D environment and hovering at a small distance above the bottom.

The authors thank Stephan Herminghaus and Christian Bahr for stimulating discussion. The DFG Priority Program 'Microswimmers' (SPP 1726) is acknowledged for funding under grants Se 1118/6, Ba 1048/8, He 2016/15 \& Ma 6330/1. Open access funding provided by Max Planck Society.

\section{References}

1. H. Tanaka, T. Araki, Phys. Rev. Lett. 81, 389 (1998)

2. F. Califano, R. Mauri, R. Shinnar, Phys. Fluids 17, 094109 (2005)

3. S. Thakur, et al., Phys. Rev. Lett. 97, 115701 (2006)

4. D. Molin, R. Mauri, V. Tricoli, Langmuir 23, 7459 (2007)

5. P. Poesio, G. Beretta, T. Thorsen, Phys. Rev. Lett. 103, 064501 (2009)

6. T. Ban, A. Aoyama, T. Matsumoto, Chem. Lett. 39, 1294 (2010)

7. T. Ban, T. Yamada, A. Aoyama, Y. Takagi, Y. Okano, Soft Matter 8, 3908 (2012)

8. J. Zhang, Y. Yao, L. Sheng, J. Liu, Advanced Materials 27, 2648 (2015)

9. S. Thutupalli, R. Seemann, S. Herminghaus, New J. Phys. 13, 073021 (2011)

10. S. Thutupalli, Towards Autonomous Soft Matter Systems (Springer, Heidelberg, 2014)

11. S. Herminghaus, et al., Soft Matter 10, 7008 (2014)

12. C.C. Maass, C. Krüger, S. Herminghaus, C. Bahr, Anu. Rev. 7, 6.1 (2016)

13. J.R. Blake, J. Fluid. Mech. 46, 199 (1971)

14. A.A. Darhuber, S.D. Trojan, Annu. Rev. Fluids 25, 061701 (2015)

15. R. Balasubramaniam, R.S. Subramanian, Phys. Fluids 12, 733 (2000)

16. R. Balasubramaniam, R.S. Subramanian, Ann. New York Acad. Sci. 1027, 303 (2004)

17. E. Guyon, et al., Physical Hydrodynamics (Oxford University Press, 2001)

18. D.A. Saville, Chem. Eng. J. 5, 251 (1973) 
19. M.D. LeVan, J. Newman, Am. Inst. Chem. Eng. J. 22, 695 (1976)

20. M.D. LeVan, J. Colloid Interface Sci. 83, 11 (1981)

21. L.E. Scriven, Chem. Eng. Sci. 12, 98 (1960)

22. S.K. Agrawal, D.T. Wasan, Chem. Eng. J. 18, 15 (1979)

23. M. Schmitt, H. Stark, Europhys. Lett. 101, 44008 (2013)

24. T. Toyota, et al., Chem. Lett. 35, 708 (2006)

25. M.M. Hanczyc, et al., J. Am. Chem. Soc. 129, 9386 (2007)

26. T. Toyota, et al., J. Am. Chem. Soc. 131, 5012 (2009)

27. T. Banno, R. Kuroha, T. Toyota, Langmuir 28, 1190 (2012)

28. T. Ban, K. Tani, H. Nakata, Y. Okano, Soft Matter 10, 6316 (2014)

29. H. Kitahata, et al., Phys. Rev. E 84, 015101(R) (2011)

30. T. Ban, T. Yamagami, H. Nakata, Y. Okano, Langmuir 29, 2554 (2013)

31. S. Miura, et al., Langmuir 30, 7977 (2014)

32. F.D. Dos Santos, T. Ondarcuhu, Phys. Rev. Lett. 75, 2972 (1995)

33. A.N. Zaikin, A.M. Zhabotinsky, Nature 225, 535 (1970)

34. A.T. Winfree, Science 175, 634 (1972)

35. S. Michelin, E. Lauga, D. Bartolo, Phys. Fluids 25, 061701 (2013)

36. N.M. Kovalchuk, D. Vollhardt, Adv. Colloid Interface Sci. 120, 1 (2006)

37. K. Nagai, Y. Sumino, H. Kitahata, K. Yoshikawa, Phys. Rev. E 7, 065301 (2005)

38. Y.J. Chen, Y. Nagamine, K. Yoshikawa, Phys. Rev. E 80, 016303 (2009)

39. Y.J. Chen, K. Yoshikawa, Eur. Phys. J. E. 34, 1 (2011)

40. K.P. Browne, et al., Angew. Chemie Int. Ed. 49, 6756 (2010)

41. V. Pimienta, et al., Angew. Chemie Int. Ed. 50, 10728 (2011)

42. A.A. Peña, C.A. Miller, Adv. Colloid Interface Sci. 123-126 241 (2006)

43. B.H. Chen, C.A. Miller, P.R. Garrett, Colloids Surfaces A 128, 129 (1997)

44. B.H. Chen, C.A. Miller, P.R. Garrett, Langmuir 31, 7463 (1998)

45. Z. Izri, et al., Phys. Rev. Lett. 113, 248302 (2014)

46. S. Michelin, E. Lauga, J. Fluid Mech. 747, 572 (2014)

47. K. Peddireddy, et al., Langmuir 28, 12426 (2012)

48. C. Krüger, C. Bahr, S. Herminghaus, C.C. Maass, Eur. Phys. J. E 39, 64 (2016)

49. S. Ariyaprakai, S.R. Dungan, Langmuir 24, 3061 (2008)

Open Access This is an Open Access article distributed under the terms of the Creative Commons Attribution License (http://creativecommons.org/licenses/by/4.0), which permits unrestricted use, distribution, and reproduction in any medium, provided the original work is properly cited. 